\title{
Surfactant protein A is expressed in the central nervous system of rats with experimental autoimmune encephalomyelitis, and suppresses inflammation in human astrocytes and microglia
}

\author{
XUE YANG, JUN YAN and JUAN FENG \\ Department of Neurology, Shengjing Hospital, Affiliated Hospital of China Medical University. \\ Shenyang, Liaoning 110004, P.R. China
}

Received October 26, 2015; Accepted February 13, 2017

DOI: $10.3892 / \mathrm{mmr} .2017 .6441$

\begin{abstract}
The collectin surfactant protein-A (SP-A), a potent host defense molecule, is well recognized for its role in the maintenance of pulmonary homeostasis and the modulation of inflammatory responses. While previous studies have detected SP-A in numerous extrapulmonary tissues, there is still a lack of information regarding its expression in central nervous system (CNS) and potential effects in neuroinflammatory diseases, such as multiple sclerosis (MS). The present study used experimental autoimmune encephalomyelitis (EAE), the most commonly used animal model of MS, to investigate the expression of SP-A in the CNS at different stages of disease progression. In addition, in vitro experiments with lipopolysaccharide (LPS)-stimulated human astrocytes and microglia were performed to investigate the potential role of SP-A in the modulation of CNS inflammatory responses. The results of the present study demonstrated widespread distribution of SP-A in the rat CNS, and also identified specific expression patterns of SP-A at different stages of EAE. In vitro, the current study revealed that treatment of human astrocytes and microglia with LPS promoted SP-A expression in a dose-dependent manner. Furthermore, exogenous SP-A protein significantly decreased Toll-like receptor 4 and nuclear factor- $\kappa \mathrm{B}$ expression, and reduced interleukin- $1 \beta$ and tumor necrosis factor- $\alpha$ levels. The results of the current study indicate a potential role for SP-A in the modulation of CNS inflammatory responses.
\end{abstract}

Correspondence to: Dr Juan Feng, Department of Neurology, Shengjing Hospital, Affiliated Hospital of China Medical University, 36 Sanhao Street, Shenyang, Liaoning 110004, P.R. China

E-mail: fengjuanneurology@yahoo.com

Abbreviations: CNS, central nervous system; SP-A, surfactant protein-A; TLR4, Toll-like receptor 4; EAE, experimental autoimmune encephalomyelitis

Key words: surfactant protein-A, experimental autoimmune encephalomyelitis, inflammation, astrocyte, microglia, Toll-like receptor 4

\section{Introduction}

Surfactant protein A (SP-A), a collagen-containing C-type collectin, is an important host defense protein that functions to maintain pulmonary homeostasis and regulate inflammatory responses $(1,2)$. SP-A is involved in various immune functions by mediating a variety of processes, including recognition and opsonization of pathogenic invaders and apoptotic cells, downregulation of inflammation and modulation of the expression of Toll-like receptors (TLRs) (3-5). During acute and chronic lung injury, serum levels of SP-A are markedly increased and SP-A knockout mice experience more severe inflammation with increased levels of pro-inflammatory cytokines $(1,6)$. Although the lung appears to be the primary site of synthesis (7), SP-A protein and mRNA are widely expressed in several extrapulmonary tissues, including the intestine, colon, mucosa and brain tissue (8-10). Previous studies have demonstrated that SP-A modulates inflammatory responses in an animal model of experimental necrotizing enterocolitis (11) and increased expression of SP-A was observed during chronic rhinosinusitis and allergic rhinitis $(12,13)$. The potential function of SP-A in extrapulmonary immunity is an emerging area of research.

Previous studies have revealed that SP-A is expressed in rat and human central nervous systems (CNS) $(10,14)$. SP-A exhibits immunoreactivity in rat and human brains, and levels are lower in the cerebrospinal fluid (CSF) of patients with autoimmune inflammatory diseases, such as multiple sclerosis (MS), which indicates that there is an association between the established functions of SP-A and the pathogenic factors of MS $(10,14)$. TLR4 is a critical cellular receptor for SP-A (15). Increased expression and activation of CNS TLR4 is involved in the activation and modulation of proinflammatory responses during the progression of MS (16). TLR4 is widely expressed in resident immune cells of the $\mathrm{CNS}$, including astrocytes and microglia, and stimulates the pathway that predominantly activates the transcription factor nuclear factor- $\kappa \mathrm{B}(\mathrm{NF}-\kappa \mathrm{B})(17,18)$. Through this pathway, TLR4 regulates the production of several inflammatory factors, including tumor necrosis factor- $\alpha$ (TNF- $\alpha)$ and interleukin-1 $\beta$ (IL-1 $\beta$ ) (19,20). Accordingly, we hypothesized that SP-A may be involved in the modulation of inflammatory 
responses in the CNS, and interact with the lipopolysaccharide (LPS)-TLR4/NF- $\mathrm{B}$ pathway in astrocytes and microglia. Using the established rat experimental autoimmune encephalomyelitis (EAE) model of MS, and in vitro experiments with LPS-stimulated human astrocytes and microglia, the present study investigated the distribution of SP-A in the rat CNS and changes in SP-A expression over different courses of EAE progression. Additionally, the current study focused on the interactions between SP-A and LPS-induced inflammatory responses, and TLR4 expression in human astrocyte and microglial cells.

\section{Materials and methods}

Animal models. Female Lewis rats $(\mathrm{n}=40)$ aged 6-8 weeks old and weighing $\sim 200 \mathrm{~g}$ were obtained from the animal laboratories of China Medical University (CMU; Shenyang, China). Animals were housed under a $12 \mathrm{~h}$ light/dark cycle and had free access to food and water. All experimental protocols were performed in accordance with the Guidelines for the Care and Use of Laboratory Animals of CMU and National Institutes of Health Publication (NIH publication no. 86-23, revised 1985). EAE model was induced as previously described (21). Briefly, rats were immunized with subcutaneous injections of $0.4 \mathrm{ml}$ solution composed of guinea pig spinal cord homogenate and an equal volume of Mycobacterium tuberculosis H37Ra (10 mg/ml; Difco; BD Biosciences, Franklin Lakes, NJ, USA)-enriched complete Freund's adjuvant (Sigma-Aldrich; Merck KGaA, Darmstadt, Germany) in both hind footpads and in the back. The day of immunization was termed day 0 . A preliminary experiment was performed prior to the detection of SP-A in different stages of EAE. It was observed that disease onset typically occurred between 9 and 11 days after immunization, and reached its peak between days 15 and 17 post-immunization. From day 21 onwards, the disease entered recovery stage. Therefore, the stages in the present study were designed to consist of initial, peak and recovery stages and the CNS tissues were obtained on day 10, day 16 and day 21 for different stages $(n=10$ per stage). Non-immunized rats $(n=10)$ received an equal amount of normal saline as a control group. All rats were sacrificed under anesthesia to obtain CNS tissue.

Preparation of purified SP-A. SP-A protein used in this study was prepared as described previously (21). Briefly, bronchoalveolar lavage was obtained from human volunteers with pulmonary alveolar proteinosis, and SP-A was isolated from lavage material by pelleting and delipidating with isopropyl and 1-butanol, the purified SP-A protein was donated by the Respiratory Department of Shenjing Hospital (Shenyang, China). Following precipitation, SP-A was further purified via column affinity and purification $(\sim 0.8 \mathrm{mg} / \mathrm{ml})$, which was determined by SDS-PAGE. Endotoxin levels in the SP-A preparation were assessed via immunoblotting and ranged from undetectable to $0.18 \mathrm{pg} / \mu \mathrm{g}$.

Human astrocyte and microglial cultures. Human astrocytes and microglia (cat. nos. 1800 and 1900, respectively; Sciencell Research Laboratories, Carlsbad, CA, USA) derived from normal human brain were cultured with a mixture of formulated Dulbecco's modified Eagle's medium (Invitrogen;
Thermo Fisher Scientific, Inc., Waltham, MA, USA) and $10 \%$ fetal bovine serum (Invitrogen; Thermo Fisher Scientific, Inc.) in a humidified atmosphere of $5 \% \mathrm{CO}_{2}$ at $37^{\circ} \mathrm{C}$. The culture medium was renewed every two days. Cells were dissociated with $0.25 \%$ EDTA (Invitrogen; Thermo Fisher Scientific, Inc.) once $80 \%$ confluency was reached.

Immunohistochemical staining for SP-A, glial fibrillary acidic protein (GFAP), allograft inflammatory factor 1 (Iba-1) and $C D 11 b$ in the rat $C N S$. Tissue specimens from rat CNS tissues were fixed in $4 \%$ paraformaldehyde (PFA) in PBS (pH 7.4) overnight and subsequently embedded in paraffin, from which $4 \mu \mathrm{m}$ thick sections were prepared. Following deparaffinization, sections were blocked with 5\% normal goat serum (Beyotime Institute of Biotechnology, Haimen, China) at room temperature for $30 \mathrm{~min}$, and incubated with rabbit SP-A polyclonal antibody (1:200; cat. no. sc-13977; Santa Cruz Biotechnology, Inc., Dallas, TX, USA), rabbit GFAP polyclonal antibody (1:500; cat. no. ab7260; Abcam, Cambridge, UK), rabbit IBA1 polyclonal antibody (1:500; cat. no. GTX101495; GeneTex, Inc., Irvine, CA, USA) or rabbit CD11b polyclonal antibody (1:200; cat. no. DF2911; Affinity Biosciences, Cincinatti, $\mathrm{OH}, \mathrm{USA}$ ) overnight at $4^{\circ} \mathrm{C}$. Subsequently, sections were incubated with goat anti-rabbit $\mathrm{IgG}(\mathrm{H}+\mathrm{L})$ secondary antibody (1:1,000; cat. no. A0277; Beyotime Institute of Biotechnology) at room temperature for $30 \mathrm{~min}$ and stained with 3,3'-diaminobenzidine (DAB) solution (Sigma-Aldrich; Merck KGaA). When detection of the distribution of SP-A in healthy rat CNS was performed, lung sections were stained as the positive control. CNS sections incubated with PBS instead of a primary antibody served as the negative control. All sections were counterstained with hematoxylin to identify nuclei. In addition, conventional hematoxylin and eosin (H\&E) staining was performed. The sections of the cortex and lung were observed at x200 and x400 magnification with a light microscope (Olympus Corporation, Tokyo, Japan).

Immunofluorescence staining for $S P$-A. Paraffin sections (3-4 $\mathrm{mm}$ ) of rat CNS were deparaffinized for double fluorescence staining for SP-A and GFAP, or SP-A and Iba-1. Subsequent to blockage with $5 \%$ normal donkey serum (Sigma-Aldrich; Merck KGaA) at room temperature for $2 \mathrm{~h}$, sections were incubated with a mixture of goat SP-A polyclonal antibody (1:200; cat. no. sc-7699; Santa Cruz Biotechnology, Inc.) and rabbit GFAP polyclonal antibody (1:500; cat. no. ab7260; Abcam), or goat SP-A polyclonal antibody and rabbit Iba-1 polyclonal antibody (1:500; cat. no. GTX101495, GeneTex, Inc.) overnight at $4^{\circ} \mathrm{C}$, followed by a mixture of Alexa Fluor ${ }^{\circledR} 488$ donkey anti-rabbit IgG (1:200; cat. no. A21206; Invitrogen; Thermo Fisher Scientific, Inc.) and Alexa Fluor ${ }^{\circledR}$ 594 donkey anti-goat IgG (1:200; cat. no. A11058; Invitrogen; Thermo Fisher Scientific, Inc.) for $2 \mathrm{~h}$ at room temperature. Hoechst 33258 was used for nuclear staining. CNS sections incubated with only secondary antibody served as negative controls for GFAP, Iba-1 and SP-A. Brain sections were observed at x400 magnification with a fluorescence microscope (Leica Microsystems GmbH, Wetzlar, Germany).

Immunocytochemical staining of astrocytes and microglia. Immunocy tochemical staining for SP-A was performed in human 
A
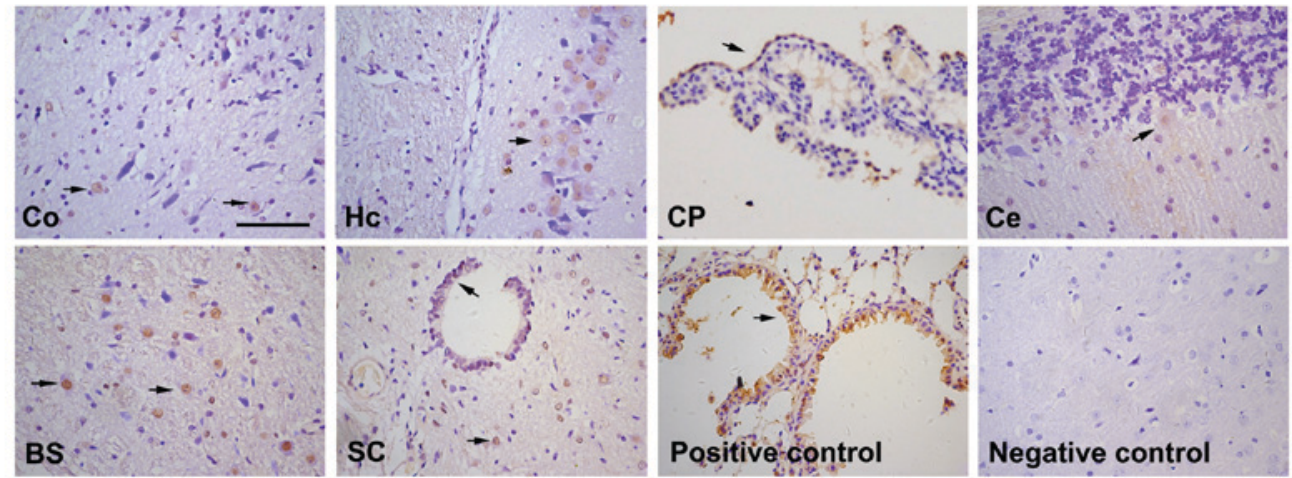

B

\section{C}

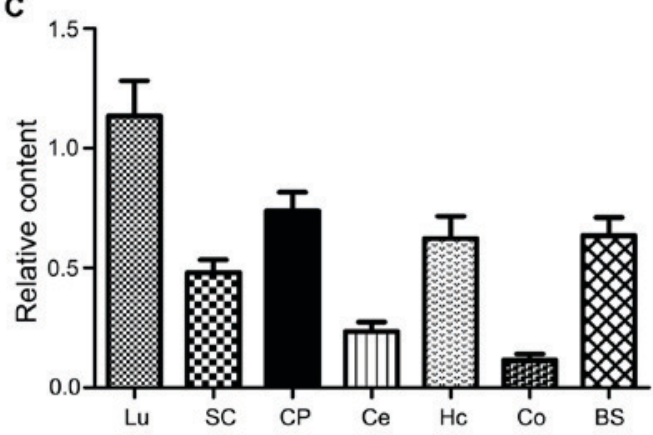

Figure 1. Detection of SP-A in rat CNS tissues by immunohistochemistry and western blotting. (A) Expression of SP-A in normal rat CNS was detected with immunohistochemistry at magnification $\mathrm{x} 400$. Sections from the $\mathrm{Co}, \mathrm{Hc}, \mathrm{CP}, \mathrm{Ce}, \mathrm{BS}$ and SC were analyzed. Lu sections from normal rats were stained as a positive control and negative control sections of normal rat brains were stained with secondary antibody only. Arrows indicate the location of SP-A expressing cells. (B) Proteins isolated from SC, CP, Hc, Co and BS were detected with western blot analysis, Lu served as a positive control. (C) Western blot quantitative analysis was performed and levels were normalized relative to $\beta$-actin. Data is presented as the mean \pm standard deviation. Co, cortex; Hc, hippocampus; $\mathrm{CP}$, choroid plexus; Ce, cerebellum; BS, brain stem; SC, spinal cord; Lu, lung; SP-A, surfactant protein-A; CNS, central nervous system.

astrocytes and microglia in vitro. Coverglass cultured cells were fixed in 4\% PFA, blocked with $5 \%$ normal goat serum for $30 \mathrm{~min}$ and incubated with rabbit SP-A polyclonal antibody (1:200; cat. no. sc-13977; Santa Cruz Biotechnology, Inc.) overnight at $4^{\circ} \mathrm{C}$, followed by rabbit $\mathrm{IgG}$ secondary antibody at room temperature for $30 \mathrm{~min}$. Sections were stained with DAB solution (Sigma-Aldrich; Merck KGaA), followed by counterstaining with hematoxylin to observe nuclei. Cells incubated only with secondary antibody served as negative controls.

Evaluation of LPS stimulation of SP-A protein expression in human astrocytes and microglia. Human astrocytes and microglia were incubated with a range of LPS concentrations from Escherichia coli 0111: B4 (0, 1, 5 and $10 \mu \mathrm{g} / \mathrm{ml}$; Sigma; St. Louis, MO, USA) for $24 \mathrm{~h}$, or $10 \mu \mathrm{g} / \mathrm{ml}$ LPS for different durations $(2,4,8,16$ and $24 \mathrm{~h})$. Cells were collected for analysis of SP-A protein expression by western blotting.

Evaluation of the effect of SP-A on LPS-induced TLR4/NF- $\kappa B$ expression and $T N F-\alpha / I L-1 \beta$ release. Human astrocytes and microglial cells were divided into the following four groups: LPS, incubated with $5 \mu \mathrm{g} / \mathrm{ml}$ LPS for $8 \mathrm{~h}$; LPS + SP-A, incubated with $5 \mu \mathrm{g} / \mathrm{ml} \mathrm{LPS}+0.5 \mu \mathrm{g} / \mathrm{ml}$ SP-A for $8 \mathrm{~h}$; SP-A, incubated with $0.5 \mu \mathrm{g} / \mathrm{ml} \mathrm{SP}-\mathrm{A}$ for $8 \mathrm{~h}$; and control, incubated with normal culture medium. Cells and culture media were collected separately for the analysis of TLR4/NF- $\mathrm{BB}$ proteins by western blotting, and TNF- $\alpha /$ IL- $1 \beta$ levels by ELISA.
Protein preparation and western blotting. Proteins from CNS tissues and cell cultures were extracted using radioimmunoprecipitation assay lysis buffer (cat. no. sc-24948; Santa Cruz Biotechnology, Inc.) and $10 \mathrm{mg} / \mathrm{ml}$ phenylmethanesulfonyl fluoride (Sigma-Aldrich; Merck KGaA) for $15 \mathrm{~min}$. Homogenates were centrifuged at $15,000 \mathrm{x} \mathrm{g}$ for $30 \mathrm{~min}$ at $4^{\circ} \mathrm{C}$, and the supernatant was collected. Protein concentrations were quantified with the BCA assay (Novagen; Merck Millipore). Protein samples (30-50 $\mu \mathrm{g} /$ well) were subjected to $10 \%$ SDS-PAGE and transferred to PVDF membranes (EMD Millipore, Billerica, MA, USA). Subsequent to blocking with 5\% skimmed milk solution at room temperature for $2 \mathrm{~h}$, membranes were incubated with rabbit SP-A polyclonal antibody (1:200; cat. no. sc-13977; Santa Cruz Biotechnology, Inc.), rabbit TLR4 polyclonal antibody (1:200; cat. no. sc-10741; Santa Cruz Biotechnology, Inc.), rabbit NF- $\mathrm{B}$ polyclonal antibody (1:200; cat. no. sc-109, Santa Cruz Biotechnology, Inc.) or mouse $\beta$-actin monoclonal antibody (1:1,000; cat. no. sc-69879, Santa Cruz Biotechnology, Inc.) overnight at $4{ }^{\circ} \mathrm{C}$, followed by peroxidase-conjugated goat anti-rabbit or anti-mouse secondary antibody (1:2,000; cat. nos. TA130023 and 130004, respectively; OriGene Technologies, Inc., Beijing, China) at room temperature for $2 \mathrm{~h}$. Immunoproducts were detected by chemiluminescence with Luminol reagent (cat.no. sc-2048; Santa Cruz Biotechnology, Inc.) and analyzed using an electrophoresis gel imaging analysis system (MF-ChemiBIS 3.2; DNR Bio-Imaging Systems, Ltd., Jerusalem, Israel). Subsequently, densitometry of the bands was 


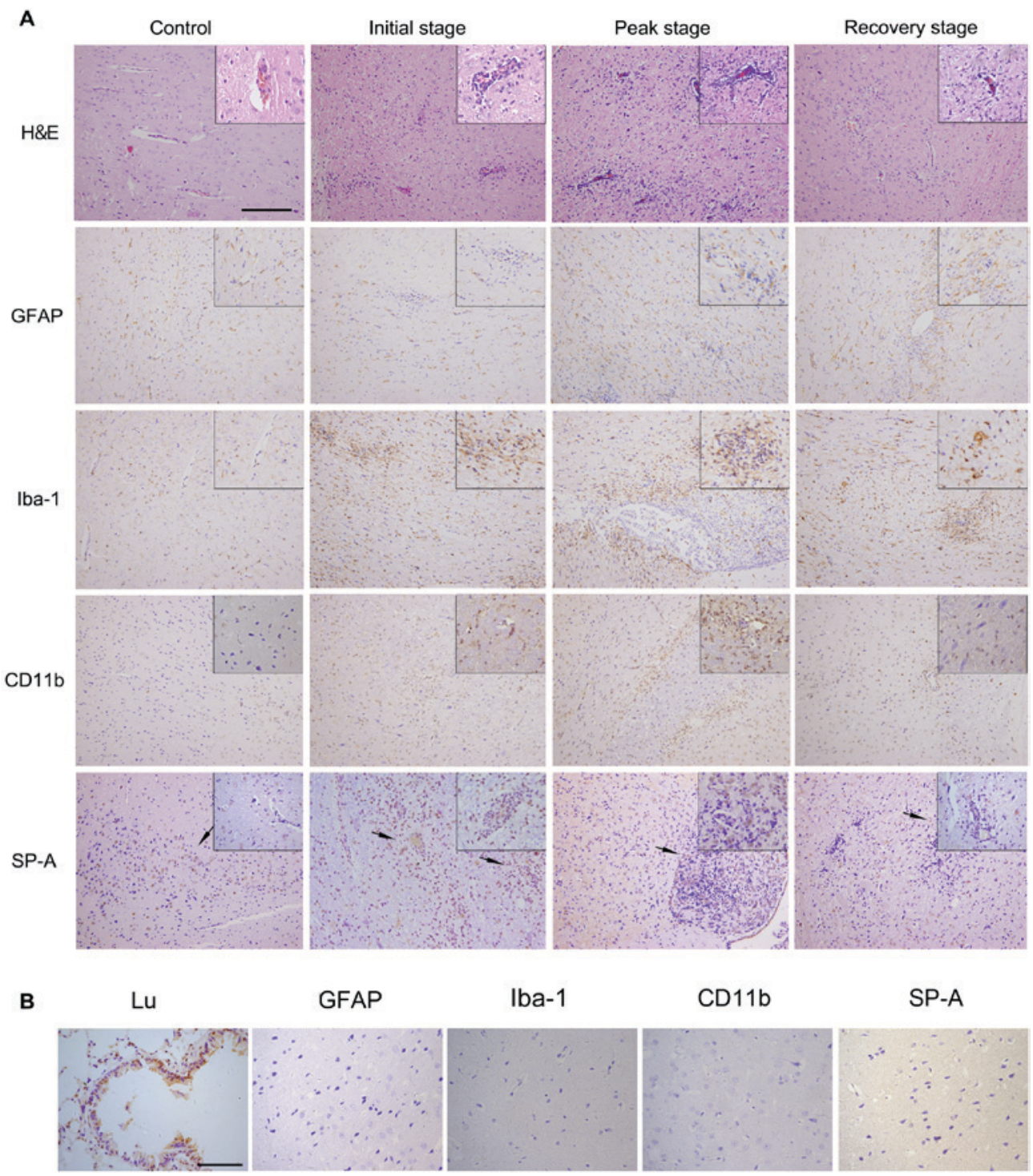

Figure 2. H\&E staining and immunohistochemical staining of GFAP, Iba-1, CD11b and SP-A in the rat CNS at different stages of EAE. (A) CNS sections at initial, peak and recovery stages of EAE were stained for inflammation by H\&E, GFAP-positive astrocytes, Iba-1-postive microglia, CD11b general marker of inflammatory cells and SP-A. Whole image magnification, x200 and corner image magnification, x400. Section of rats under normal conditions served as the control. Arrows indicate the location of SP-A expressing cells. (B) Lu sections of normal rats were stained as a positive control. Negative control sections of normal rat CNS were treated with secondary antibody only and were unstained for GFAP, Iba-1, CD11b and SP-A. Magnification, x400. H\&E, hematoxylin and eosin; GFAP, glial fibrillary acidic protein; Iba-1, ionized calcium-binding adapter molecule 1; SP-A, surfactant protein-A; Lu, lung; CNS, central nervous system; EAE, experimental autoimmune encephalomyelitis.

semi-quantitatively analyzed (n=9 per group) using Image-Pro Plus 6.0 software (Media Cybernetics, Inc., Rockville, MD, USA) and calculated relative to $\beta$-actin control.

ELISA analysis of TNF- $\alpha$ and $I L-1 \beta$ release. Culture medium from the 4 groups was centrifuged at $15,000 \times \mathrm{g}$ for $30 \mathrm{~min}$ to remove debris. The supernatant was collected and used to analyze TNF- $\alpha$ and IL- $1 \beta$ levels using matched ELISA kits (cat. nos. DTA00C and DLB50, respectively; R\&D systems, Inc., Minneapolis, MN, USA). Concentrations were quantified relative to standard curves, according to the manufacturer's instructions.

Statistical analysis. All data were analyzed using GraphPad Prism 6.0 software (GraphPad Software, Inc., La Jolla,
CA, USA) and presented as the mean \pm standard deviation. Differences among groups were analyzed by one-way analysis of variance, followed by the least significant difference test or Kruskal-Wallis test. $\mathrm{P}<0.05$ was considered to indicate a statistically significant difference.

\section{Results}

Detection of SP-A in healthy rat CNS tissues. SP-A was detected in brain and spinal cord tissue sections from normal rats via immunostaining. Immunohistochemical results demonstrated widespread distribution of SP-A. In the cortex, SP-A was demonstrated to be expressed on glial cells rather than neurons and in positively stained cells the staining intensity was higher in the nuclei than in the cytoplasm (Fig. 1A). SP-A 
A

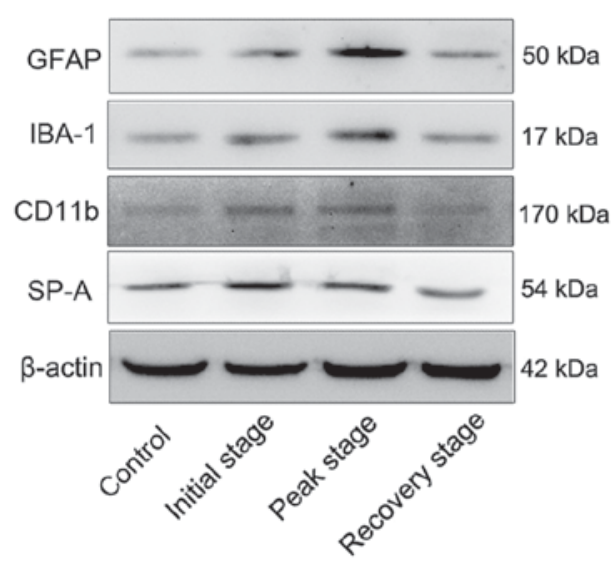

B

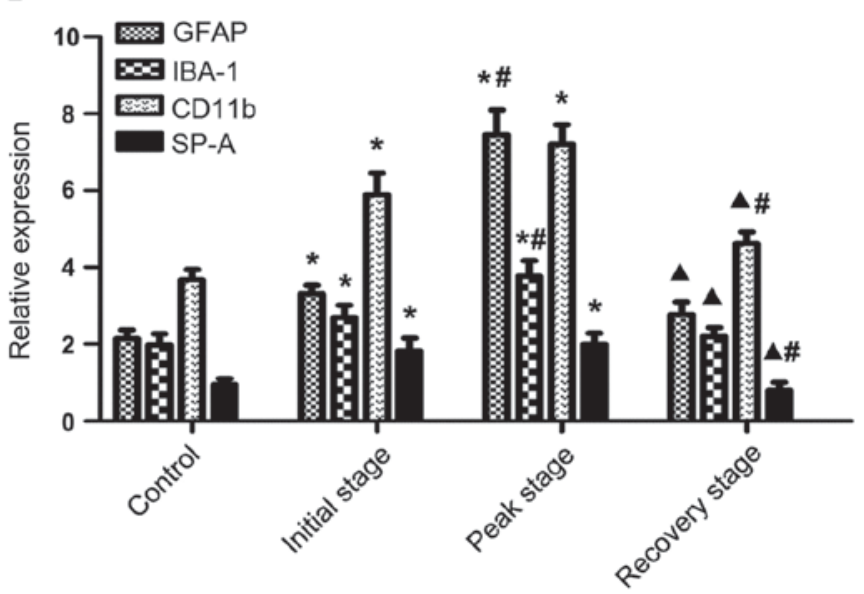

Figure 3. Western blot analysis of GFAP, Iba-1, CD11b and SP-A in the rat CNS at different stages of EAE. (A) Proteins obtained from the rat CNS at the initial, peak and recovery stages of EAE were detected for GFAP, IBA-1, CD11b and SP-A via western blotting. (B) Quantitative analysis of western blots was performed and levels were normalized relative to $\beta$-actin. Results are presented as the mean \pm standard deviation. ${ }^{*} \mathrm{P}<0.05$ vs. control group; ${ }^{\sharp} \mathrm{P}<0.05$ vs. initial stage group; ${ }^{\wedge} \mathrm{P}<0.05$ vs. peak stage group. GFAP, glial fibrillary acidic protein; Iba-1, ionized calcium-binding adapter molecule 1; SP-A, surfactant protein-A; CNS, central nervous system; EAE, experimental autoimmune encephalomyelitis.

was also expressed in the cytoplasm and granular structures of hippocampal neurons. Strong immunoreactivity for SP-A was detected in the cytoplasm of ependymocytes of the outer layer of choroid plexus. SP-A was also present in Purkinje cells of the cerebellar cortex. In a vertical section of brain stem and horizontal section of spinal cord, the nuclei and cytoplasm of specific cells, presumably glia, exhibited immunoreactivity for SP-A. Lung tissue sections, used as a positive control, exhibited positive immunoreactivity, and CNS sections incubated with only secondary antibody, used as the negative control, exhibited no immunoreactivity (Fig. 1A). Proteins isolated from spinal cord, choroid plexus, cerebellum, hippocampus, cortex and brain stem tissue were analyzed by western blotting to investigate the protein expression of SP-A, with lung tissue used as the positive control. SP-A bands from western blot analysis of different rat CNS samples are presented in Fig. 1B. The results demonstrated that choroid plexus, hippocampus and brain stem tissues expressed higher levels of SP-A protein compared with the other tissues in the rat CNS (Fig. 1C).

$H \& E$ staining and changes in the expression of SP-A, GFAP, Iba-1 and CDI1b in the initial, peak and recovery stages of $E A E$. As immunoreactivity for SP-A was detected in healthy rat CNS sections, the present study also investigated SP-A expression patterns at different stages of EAE. Parallel sections were stained for inflammation (H\&E), astrocytes (GFAP), microglia and monocytes (Iba-1), and also a general marker for inflammatory cells (CD11b), which allowed changes in SP-A expression and the degree of inflammation during EAE to be analyzed. Rats under normal conditions, with no infiltrating inflammatory cells in the brain detected by HE staining and normal microglial/leukocyte CD11b expression, were used as the control. GFAP-positive astrocytes and Iba-1 positive microglia were evenly distributed in the brain with normal morphology(Fig. 2A). At the initial stage of EAE (days 9-11), H\&E staining revealed a small number of scattered infiltrating cells around the vessels. The pattern of
GFAP-positive astrocytes was similar in the initial stage of EAE compared with the pattern in normal conditions, while Iba-1-positive microglia exhibited a modified rod shape and increased numbers in the initial stage of EAE compared with normal conditions, which was associated with inflammatory cell aggregation. Expression of CD11b was increased in peri-foci during the initial stage compared with normal conditions. In the initial stage of EAE, SP-A levels were marginally increased compared with normal conditions. Notably, immunoreactive SP-A aggregated to inflammatory foci, which is evident from immunostaining (Fig. 2A).

At the peak EAE stage (days 15 and 17), large-scale inflammatory cell aggregation and numerous perivascular cuffs were observed in HE-stained sections. Astrocytic reactions occurred around the EAE lesions with increased staining intensity. Microglial cells increased in number and were of the activated form (Fig. 2A). Microglial/leukocyte CD11b levels were also markedly increased at the peak stage compared with the initial stage and normal conditions. In CNS samples in the peak EAE stage, SP-A levels were markedly increased compared with the initial stage and normal conditions, particularly within certain severe lesions with huge amounts of inflammatory cells (Fig. 2A). From day 21 onwards, the recovery stage commenced and the number of inflammatory cells and EAE lesions observed from H\&E staining was markedly reduced compared with the peak stage. Additionally, reactive astrocytes and microglia cells decreased in number and interacted to form glial scars. Positive staining for CD11b was also decreased, along with SP-A expression in recovery stage CNS samples compared with peak stage samples. SP-A was distributed far from lesions, which was also observed in control samples (Fig. 2A). Lung sections of normal rats were stained as a positive control. Negative control sections of normal rat CNS were treated with secondary antibody only and were unstained for GFAP, Iba-1, CD11b and SP-A, as presented in Fig. 2B. Protein levels of the aforementioned indicators at different EAE stages were assessed by western 


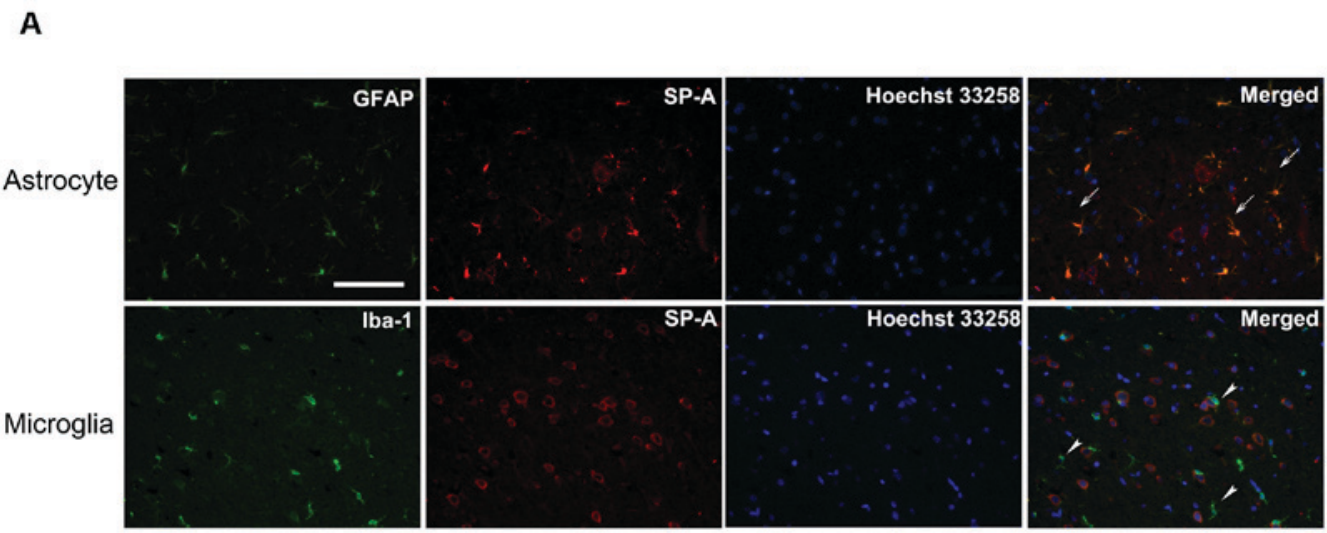

B
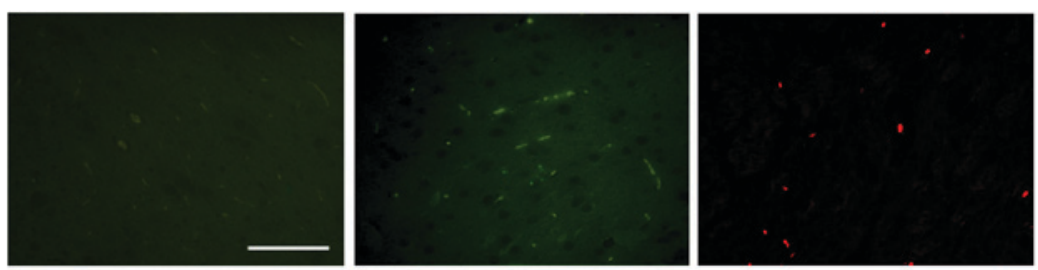

Figure 4. Double-immunofluorescence staining for detection of SP-A on astrocytes and microglia cells in the rat central nervous system. (A) Sections were immunostained with anti-GFAP and anti-SP-A, or anti-Iba-1 and anti-SP-A antibodies. Nuclei were counterstained with Hoechst 33258. GFAP+/SP-A+ astrocytes are indicated by arrows and Iba-1+/SP-A- microglial cells are indicated by arrowheads in the merged images. Magnification, x400. (B) Negative control sections treated with secondary antibody only were unstained for GFAP, Iba-1 and SP-A. Magnification, x400. GFAP, glial fibrillary acidic protein; SP-A, surfactant protein-A; Iba-1, ionized calcium-binding adapter molecule 1.
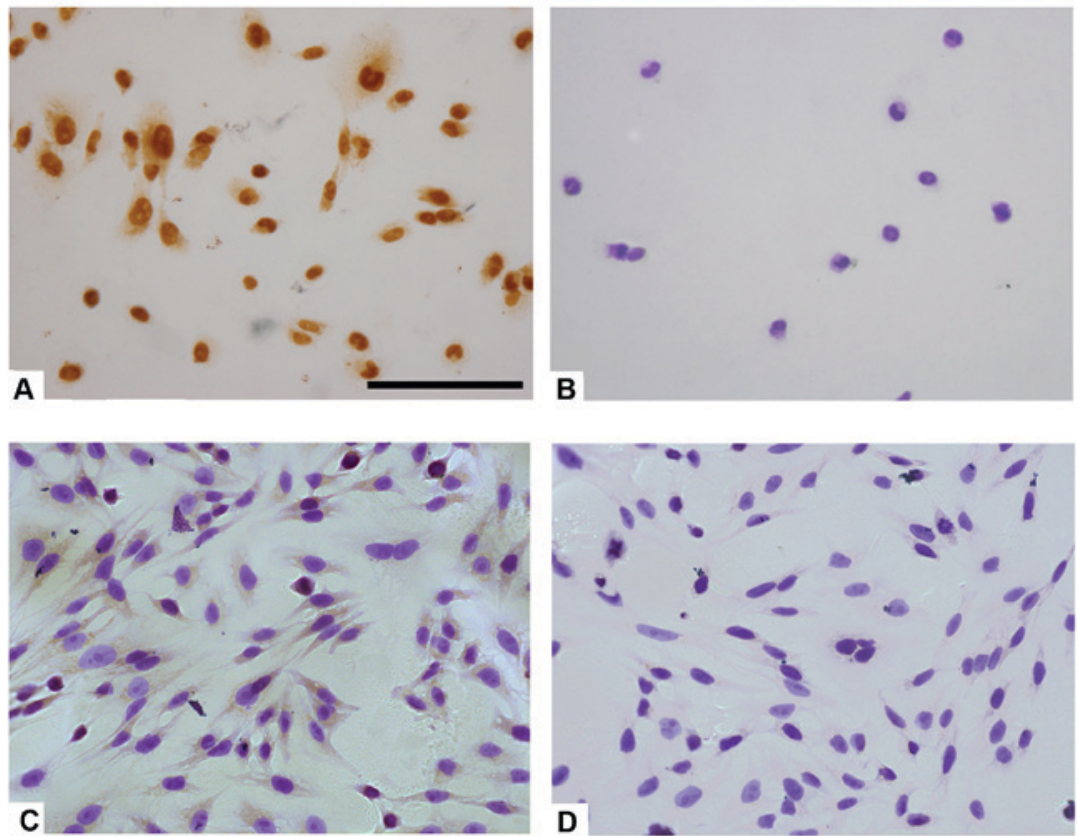

Figure 5. Immunohistochemical staining of SP-A in human astrocytes and microglial cells. Fixed human astrocytes were stained for (A) SP-A or (B) with secondary antibody only. Fixed human microglial cells were stained for (C) SP-A or (D) with secondary antibody only. Magnification, x400. All sections were counterstained with hematoxylin for nuclei. Immunopositive SP-A was detected in the cytoplasm and nuclei of human astrocytes, however, only in the cytoplasm of microglia. SP-A, surfactant protein-A.

blot analysis (Fig. 3A). Quantification of blots demonstrated gradually increasing expression of GFAP, Iba-1 and $\mathrm{CD} 11 \mathrm{~b}$ in the initial and peak stages compared with control samples, followed by a significant decrease in expression at the recovery stage compared with the peak stage $(\mathrm{P}<0.05$; Fig. 3B). Similarly, SP-A protein expression increased with 
A

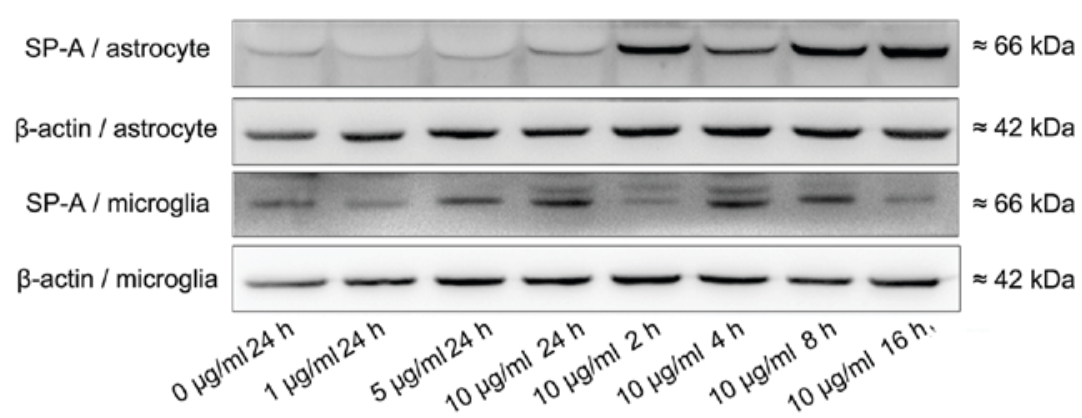

B

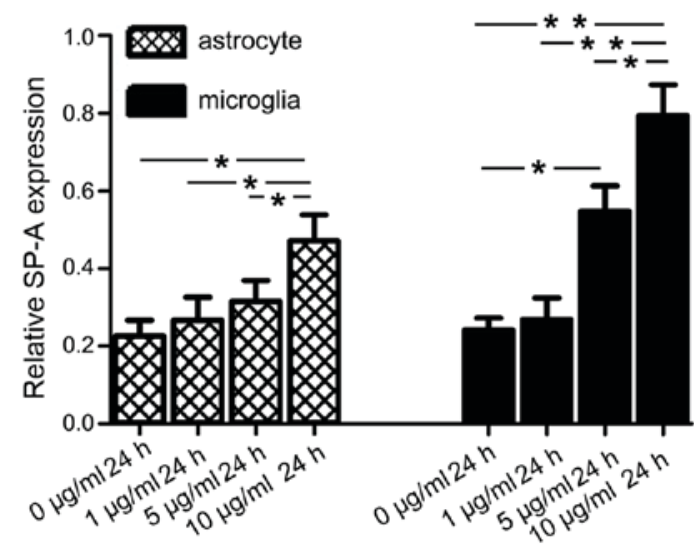

C

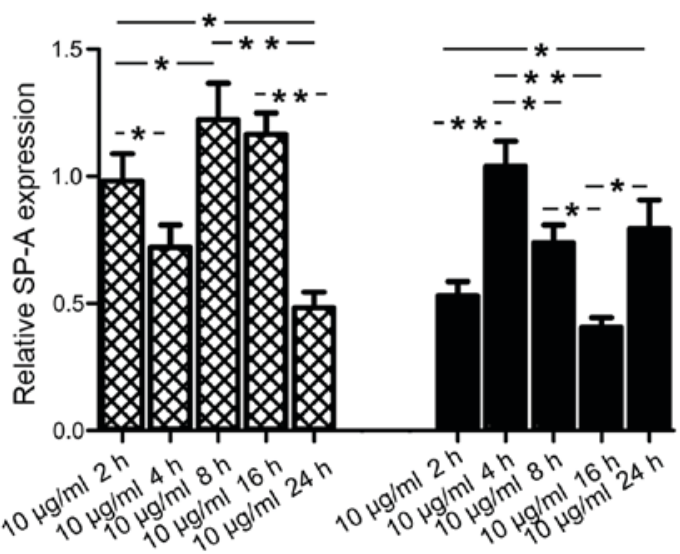

Figure 6. SP-A protein levels are increased in LPS-stimulated human astrocytes and microglial cells. Human astrocytes and microglia cells were stimulated with either varying doses of LPS $(0,1,5$, and $10 \mu \mathrm{g} / \mathrm{ml})$ for $24 \mathrm{~h}$ or $10 \mu \mathrm{g} / \mathrm{ml}$ LPS for different durations $(2,4,8,16$ or $24 \mathrm{~h})$. (A) SP-A protein levels isolated from cultured cells were assessed by western blot analysis. Quantitative analysis of western blotting results from (B) varying LPS doses and (C) varying durations of exposure to $10 \mu \mathrm{g} / \mathrm{ml}$ LPS were normalized relative to $\beta$-actin. Results are presented as the mean \pm standard deviation. ${ }^{*} \mathrm{P}<0.05$ and ${ }^{* *} \mathrm{P}<0.01$, comparisons indicated by brackets. SP-A, surfactant protein-A; LPS, lipopolysaccharide.

increasing severity of inflammation and expression of reactive astrocytes and microglial cells, and decreased significantly in the recovery stage compared with initial and peak stages $(\mathrm{P}<0.05$; Fig. 3B).

Detection of SP-A in astrocytes and microglia. To clarify the association between immunoreactive SP-A, and astrocytes and microglia, the two types of CNS resident immune cells, the present study detected SP-A expression in astrocytes and microglia via double immunofluorescence staining of normal rat CNS sections and immunocytochemical staining of human astrocyte and microglia cell lines. In rat CNS sections, SP-A was diffusely distributed in the cytoplasm of astrocytes, but not microglia (Fig. 4). Additionally, cytoplasmic and nuclear staining patterns of SP-A in human astrocytes were observed (Fig. 5A). Fig. 5B presents results from human astrocytes treated with secondary antibody only as a negative control. However, immunopositive SP-A was detected in the cytoplasm of human microglia cells, which contrasted to the results obtained with rat resident microglia cells (Fig. 5C). Fig. 5D presents results from human microglia treated with secondary antibody only as a negative control.
Inflammatory stimuli affect SP-A protein expression in human astrocytes and microglia. Having demonstrated that SP-A is expressed in human astrocytes and microglia, the present study subsequently investigated whether SP-A expression in these cells is affected by proinflammatory stimuli, such as LPS. Human astrocytes and microglia were exposed to a range of LPS concentrations, $(0-10 \mu \mathrm{g} / \mathrm{ml})$ for $24 \mathrm{~h}$ or $10 \mu \mathrm{g} / \mathrm{ml}$ LPS for 2-24 h, and SP-A protein levels were investigated by western blotting (Fig. 6A) and quantification was performed (Fig. 6B). After $24 \mathrm{~h}$ of exposure to $0,1,5$ or $10 \mu \mathrm{g} / \mathrm{ml} \mathrm{LPS}$, SP-A levels in both cell types increased in a dose-dependent manner $(\mathrm{P}<0.05$; Fig. 6B). However, application of $10 \mu \mathrm{g} / \mathrm{ml}$ LPS for 2, 4, 8, 16 and $24 \mathrm{~h}$ did not lead to a time-dependent increase in SP-A levels (Fig. 6C).

SP-A suppresses LPS-induced expression of TLR4 and NF- $\kappa B$ p65 in human astrocytes and microglia. To determine the potential role of SP-A in the TLR4/NF- $\kappa$ B signaling pathway, human astrocytes and microglia were treated with LPS to induce TLR signaling. LPS stimulation for $24 \mathrm{~h}$ induced

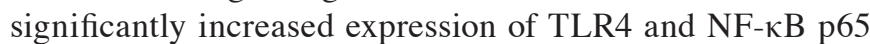
in both cell types compared with the control group $(\mathrm{P}<0.05$; 

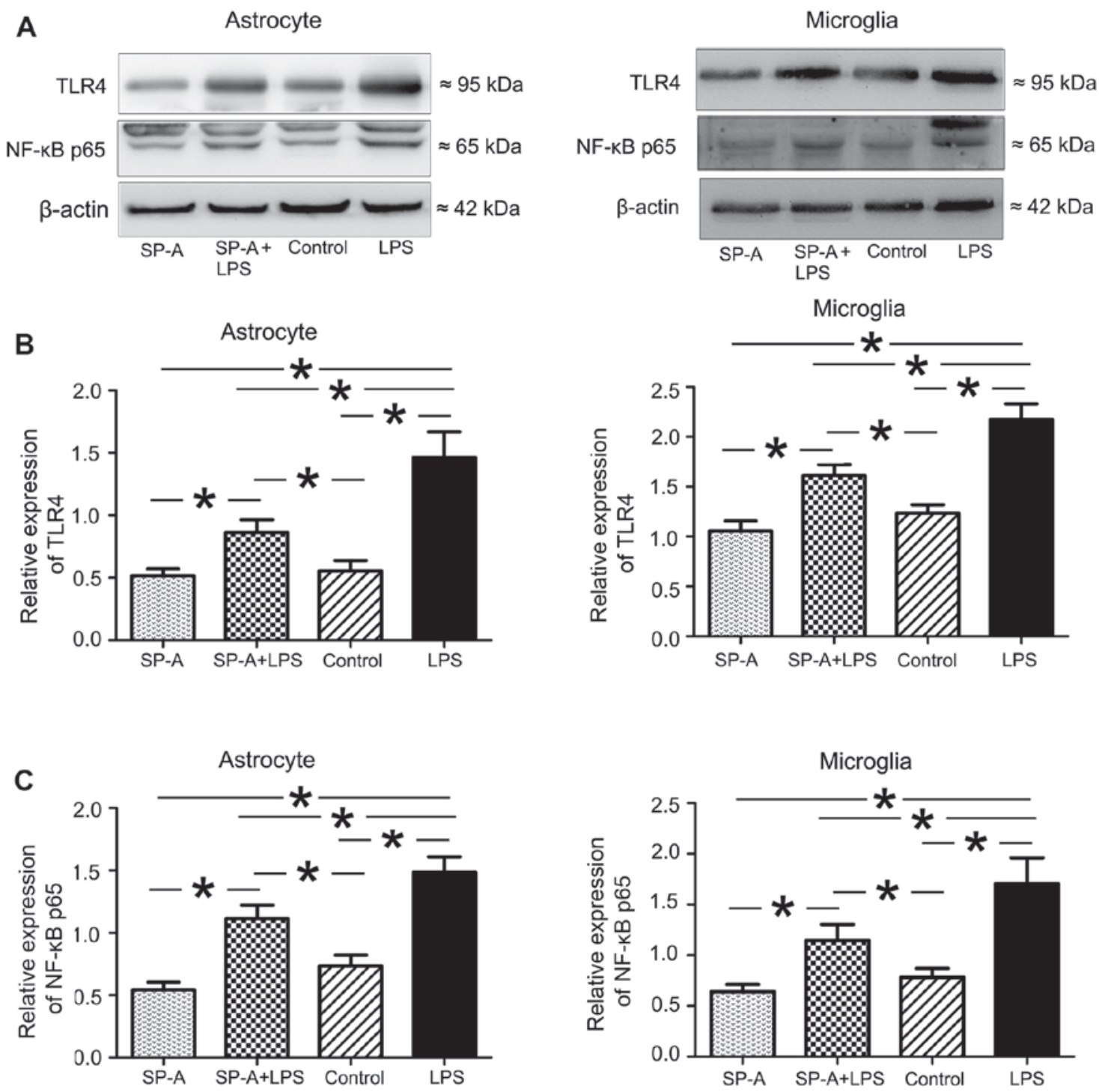

Figure 7. Effect of exogenous SP-A on the levels of TLR4 and NF- $\mathrm{kB}$ in LPS-stimulated human astrocytes and microglia. (A) Levels of TLR4 and NF-kB p65 proteins in human astrocytes and microglia were determined by western blot analysis. SP-A, cells treated with $0.5 \mu \mathrm{g} / \mathrm{ml}$ SP-A only; SP-A + LPS, cells treated with $0.5 \mu \mathrm{g} / \mathrm{ml}$ SP-A $+5 \mu \mathrm{g} / \mathrm{ml}$ LPS; control, untreated group; LPS, cells treated with $5 \mu \mathrm{g} / \mathrm{ml}$ LPS only. Densitometric quantification of (B) TLR4 and (C) NF-kB p65 relative to $\beta$-actin in human astrocytes and microglia. "P<0.05, comparisons indicated by brackets. TLR4, Toll-like receptor 4; NF- $\mathrm{kB}$, nuclear factor-кB; SP-A, surfactant protein-A; LPS, lipopolysaccharide.

Fig. 7). SP-A + LPS treatment induced a significant inhibitory effect on LPS-induced TLR4 and NF- $\mathrm{B}$ in astrocytes and microglia $(\mathrm{P}<0.05$; Fig. 7). SP-A alone reduced the expression of TLR4 and NF- $\kappa \mathrm{B}$ marginally compared with the control, however differences were not statistically significant (Fig. 7).

SP-A suppresses the release of LPS-induced TNF- $\alpha$ and IL- $1 \beta$. Increased production of proinflammatory cytokines is associated with the pathogenesis of MS and EAE. Accordingly, the present study investigated the effects of exogenous SP-A on the release of TNF- $\alpha$ and IL-1 $\beta$ in LPS-stimulated human astrocytes and microglia. As presented in Fig. 8, TNF- $\alpha$ and IL-1 $\beta$ levels in culture medium of both cell types were significantly increased in LPS groups, compared with the control groups $(\mathrm{P}<0.05)$. SP-A + LPS groups exhibited significantly decreased TNF- $\alpha$ and IL-1 $\beta$ levels in microglia supernatant, compared with the LPS groups $(\mathrm{P}<0.05$; Fig. 8). TNF- $\alpha$ and IL-1 $\beta$ levels in the
SP-A + LPS group in astrocytes were reduced compared with the LPS group, however, the reduction in TNF- $\alpha$ release was not statistically significant (Fig. 8). SP-A alone did not significantly reduce the release of TNF- $\alpha$ and IL-1 $\beta$ in astrocytes and microglia compared with the control (Fig. 8).

\section{Discussion}

The present study indicated a potential role for SP-A in the regulation of inflammatory responses in the CNS. SP-A was demonstrated to be widely distributed in rat CNS tissues and was expressed in rat and human astrocytes, and also human microglia. Changes in the expression of SP-A at different stages of EAE were also observed. The results of the current study demonstrated that LPS enhances SP-A protein expression in human astrocytes and microglial cells in a dose-dependent manner. Notably, SP-A inhibited LPS-induced expression of 

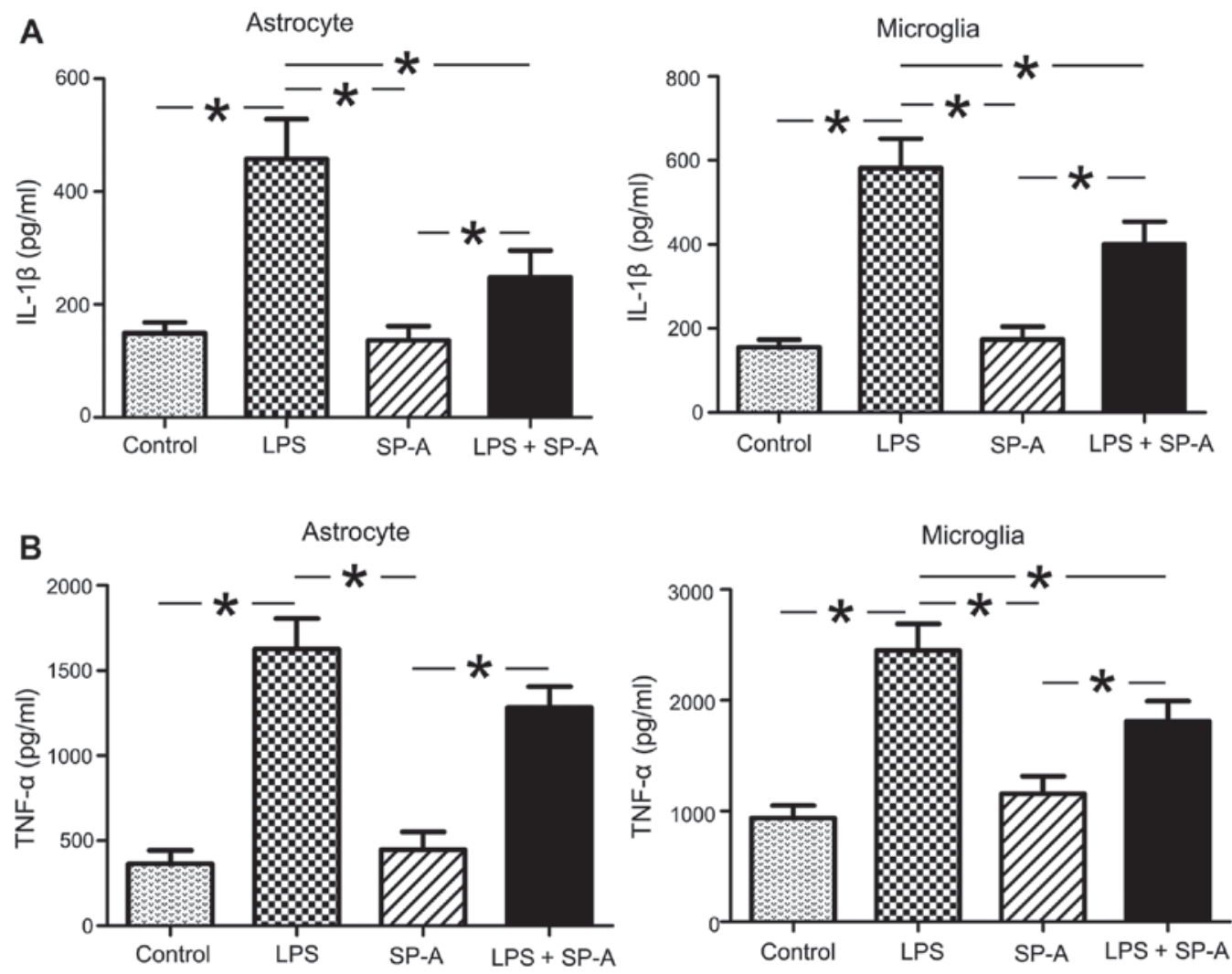

Figure 8. Effect of exogenous SP-A on IL-1 $\beta$ and TNF- $\alpha$ cytokine levels in LPS-stimulated human astrocytes and microglia. Levels of IL-1 $\beta$ (A) and TNF- $\alpha$ (B) in the culture medium of astrocytes and microglia stimulated with $5 \mu \mathrm{g} / \mathrm{ml} \mathrm{LPS}$ and $/$ or $0.5 \mu \mathrm{g} / \mathrm{ml}$ SP-A were quantified by ELISA. Results are presented as the mean \pm standard deviation. "P<0 05 , comparisons indicated by brackets. IL-1 $\beta$, interleukin-1 $\beta$; LPS, lipopolysaccharide; SP-A, surfactant protein-A; TNF- $\alpha$, tumor necrosis factor- $\alpha$.

TLR4 and NF- $\kappa B$, and LPS-induced release of TNF- $\alpha$ and IL-1 $\beta$. These results indicate that SP-A may function in the modulation of neuroinflammation, potentially through its inhibitory effects on TLR4/NF- $\mathrm{BB}$ activation and proinflammatory cytokine release.

The SP-A protein is a hydrophilic, collagen-containing lectin with a hexameric structure formed by six trimers of glycoprotein monomers. Characterization studies have suggested that this potent innate immune molecule performs a diverse range of functions, including anti-microbial activity, clearance of apoptotic and necrotic cells and control of inflammation triggered by self or non-self (22). Initially identified in the alveolar surface of the lung, SP-A was subsequently demonstrated to be widely distributed in other tissues (23), such as brain tissue (10). Schob et al (10) were the first to report an association between SP-A and CNS pathology by examining the expression patterns of SP-A in human brains, which revealed increased SP-A expression in the CSF of patients with autoimmune infectious diseases and indicated a potential role for SP-A in MS development. MS is a chronic autoimmune-mediated inflammatory disease of CNS. A primary mechanism underlying the pathogenesis of MS is that peripherally activated immune cells infiltrate the CNS, where they trigger inflammatory reactions and cause myelin damage (24). Accordingly, the present study investigated the expression of SP-A in different stages of EAE, the most widely used animal model of MS. In normal rats, SP-A was widely distributed in the CNS and expressed on various resident cells, including hippocampal neurons, cerebellum Purkinje cells, ependymal cells and certain glial cells, which indicates potential interactions between the peripheral innate and CNS immune systems. SP-A protein expression in different stages of the EAE model was subsequently examined. SP-A expression was increased as EAE progressed, and expression moved towards the inflammatory foci, as with reactive microglia cells and astrocytes. As CNS resident immune cells, astrocytes and microglia are involved in the modulation of immuno-inflammatory responses in EAE. In the present study, microglial cells responded to inflammation earlier than astrocytes. However, levels of SP-A were not markedly increased until the peak stage, consistent with the changes in expression observed in astrocytes. To further investigate the association between SP-A and astrocytes and microglia, expression of SP-A was detected in rat brain astrocytes and microglia in vivo. SP-A was demonstrated to be expressed on astrocytes but not microglia in rat brains. Notably, similar examination of SP-A expression in human astrocytes and microglia in vitro revealed that both cell lines endogenously produced and expressed SP-A protein. Furthermore, human astrocytes and microglia responded to inflammatory stimulation with LPS through dose-dependent increased SP-A expression.

In EAE, invading immune cells produce endotoxin and proinflammatory mediators, which are important triggers of CNS infection. The severity of EAE at different stages partially depends on the levels of these pathogenic substances (25). Thus, 
changes in SP-A expression in human astrocytes and microglia depend on the amount of LPS, but not time of exposure to LPS, which is consistent with in vivo results. Activation of astrocytes and microglia is reported to cause severe inflammatory insult driven by activation of the TLR4 signaling pathway via release of proinflammatory cytokines (26-28). As SP-A is an endogenous ligand for TLR4 (3), we hypothesized that the protein modulates inflammation through effects on TLR4/NF- $\mathrm{KB}$ signaling in astrocytes and microglia. Consistent with this theory, SP-A exerted a significant inhibitory effect on LPS-induced overexpression of TLR4 and NF- $\mathrm{KB}$ in both cell types. In human lungs, SP-A also regulates TLR4 activation and expression in macrophages and dendritic cells $(15,29)$. Furthermore, SP-A has been previously reported to inhibit TLR4 signaling, decrease IкB $\alpha$ phosphorylation and nuclear translocation of p65, which are critical for NF- $\mathrm{KB}$ activation, and lead to diminished TLR ligand-induced TNF- $\alpha$ secretion (15). This effect of SP-A on human astrocytes and microglia was confirmed in the current study, which demonstrated that LPS-induced secretion of TNF- $\alpha$ and IL- $1 \beta$ was suppressed in the presence of SP-A. Based on these results, LPS may promote the expression of SP-A, which may subsequently participate in the regulation of LPS-induced TLR4/NF- $\mathrm{BB}$ activation and proinflammatory cytokine release. The ability of SP-A to reduce levels of proinflammatory cytokines has been demonstrated in various mammalian diseases. Quintanilla et al (11) demonstrated that SP-A treatment of experimental necrotizing enterocolitis significantly suppressed TLR4 expression and reduced TNF- $\alpha$ and IL-1 $\beta$ levels. Retinal SP-A is also reported to localize on GFAP-reactive astrocytes in mice and TLR4 ligands upregulate SP-A expression and myeloid differentiation primary response 88 protein essential for NF- $\kappa$ B signaling (30). Similarly, SP-A has been demonstrated to significantly inhibit TLR ligand-induced expression of proinflammatory mediators, including TNF- $\alpha$ and IL-1 $\beta$, in an inflammation-induced preterm delivery model (31). The functional mechanism of SP-A-mediated regulation of CNS inflammation may be complex as SP-A has been reported to bind LPS and modulate TLR4. In the present study, SP-A potentially interacts with TLR4 directly to inhibit its overexpression and activation, alternatively, SP-A may interact with LPS to prevent it binding to TLR4 (32). Further in vitro studies using blockers or inhibitors are required to clarify this. SP-A has also been identified as an endogenous ligand for one or more receptors, including TLR2, CD14 and glycoprotein 340, each of which is thought to trigger a proinflammatory signal transduction cascade $(3,33,34)$. Thus, LPS-induced increased expression of SP-A in astrocytes and microglia may partially act on the feedback mechanism to inhibit activation of inflammatory signaling pathways induced by these receptors. Notably, these results are consistent with results from the present study. The involvement of a pulmonary innate immune protein in the regulation of CNS inflammatory pathology is not surprising. However, the contribution of SP-A in EAE is remains unclear and it is important to note that species differences exist between rats and humans with regard to the immune system. Further studies of SP-A gene deletion or overexpression in vivo are therefore required to ascertain the effects of SP-A on the regulation of CNS inflammation and also MS pathogenesis, including effects on the clearance of pathogenic immune cells and regulation of T-cell polarization.

In conclusion, the present study provides preliminary evidence of SP-A expression in normal and EAE rat CNS, and human astrocytes and microglia. The results indicate that LPS induces increased expression of SP-A protein in human astrocytes and microglia in a dose-dependent manner. Furthermore, SP-A may regulate inflammation in these cell lines at least partially by reducing the expression of TLR4 protein and inflammatory cytokine release, indicating that the immunomodulatory properties of SP-A may function in neuroinflammatory disease.

\section{Acknowledgements}

The study was partially financially supported by grants from the Liaoning Province Science and Technology Project-Animal Scientific Research and Clinical Application for Major Disease of Liaoning Province (grant no. 2012225021), the Program of Basic and Clinical Research Platform of China Medical University (grant no. CMU-201406) and the Technology Projects of Liaoning Province (grant no. 2009225010-2) awarded to Dr Juan Feng.

\section{Refrences}

1. Wright JR: Immunoregulatory functions of surfactant proteins. Nat Rev Immunol 5: 58-68, 2005.

2. Chroneos ZC, Midde K, Sever-Chroneos Z and Jagannath C: Pulmonary surfactant and tuberculosis. Tuberculosis (Edinb) 89 (Suppl 1): S10-S14, 2009.

3. Kishore U, Greenhough TJ, Waters P, Shrive AK, Ghai R, Kamran MF, Bernal AL, Reid KB, Madan T and Chakraborty T: Surfactant proteins SP-A and SP-D: Structure, function and receptors. Mol Immunol 43: 1293-1315, 2006.

4. Schaub B, Westlake RM, He H, Arestides R, Haley KJ, Campo M, Velasco G, Bellou A, Hawgood S, Poulain FR, et al: Surfactant protein D deficiency influences allergic immune responses. Clin Exp Allergy 34: 1819-1826, 2004.

5. Hartshorn KL, Crouch E, White MR, Colamussi ML, Kakkanatt A, Tauber B, Shepherd V and Sastry KN: Pulmonary surfactant proteins $A$ and D enhance neutrophil uptake of bacteria. Am J Physiol 274: L958-L969, 1998.

6. Chuang CY, Chen TL and Chen RM: Molecular mechanisms of lipopolysaccharide-caused induction of surfactant protein-A gene expression in human alveolar epithelial A549 cells. Toxicol Lett 191: 132-139, 2009.

7. Yuan HT, Gowan S, Kelly FJ and Bingle CD: Cloning of guinea pig surfactant protein A defines a distinct cellular distribution pattern within the lung. Am J Physiol 273: L900-L906, 1997.

8. Rubio S, Lacaze-Masmonteil T, Chailley-Heu B, Kahn A, Bourbon JR and Ducroc R: Pulmonary surfactant protein A (SP-A) is expressed by epithelial cells of small and large intestine. J Biol Chem 270: 12162-12169, 1995.

9. Snyder GD, Oberley-Deegan RE, Goss KL, Romig-Martin SA, Stoll LL, Snyder JM and Weintraub NL: Surfactant protein D is expressed and modulates inflammatory responses in human coronary artery smooth muscle cells. Am J Physiol Heart Circ Physiol 294: H2053-H2059, 2008.

10. Schob S, Schicht M, Sel S, Stiller D, Kekulé AS, Paulsen F, Maronde $\mathrm{E}$ and Bräuer L: The detection of surfactant proteins $\mathrm{a}, \mathrm{b}, \mathrm{c}$ and $\mathrm{d}$ in the human brain and their regulation in cerebral infarction, autoimmune conditions and infections of the CNS. PLoS One 8: e74412, 2013.

11. Quintanilla HD, Liu Y, Fatheree NY, Atkins CL, Hashmi SS, Floros J, McCormack FX, Rhoads JM and Alcorn JL: Oral administration of surfactant protein-a reduces pathology in an experimental model of necrotizing enterocolitis. J Pediatr Gastroenterol Nutr 60: 613-620, 2015.

12. Lee HM, Kang HJ, Woo JS, Chae SW, Lee SH and Hwang SJ: Upregulation of surfactant protein a in chronic rhinosinusitis. Laryngoscope 116: 328-330, 2006 
13. Wootten CT, Labadie RF, Chen A and Lane KF: Differential expression of surfactant protein A in the nasal mucosa of patients with allergy symptoms. Arch Otolaryngol Head Neck Surg 132: 1001-1007, 2006.

14. Luo JM, Wan YS, Liu ZQ, Wang GR, Floros J and Zhou HH Regularity of distribution of immunoreactive pulmonary surfactant protein A in rat tissues. Int J Mol Med 14: 343-351, 2004.

15. Henning LN, Azad AK, Parsa KV, Crowther JE, Tridandapani S and Schlesinger LS: Pulmonary surfactant protein a regulates TLR expression and activity in human macrophages. J Immunol 180: 7847-7858, 2008.

16. Duperray A, Barbe D, Raguenez G, Weksler BB, Romero IA Couraud PO, Perron $\mathrm{H}$ and Marche PN: Inflammatory response of endothelial cells to an endogenous retrovirus associated to MS is mediated by TLR4. Int Immunol 27: 545-553, 2015.

17. Parajuli B, Sonobe Y, Kawanokuchi J,Doi Y,Noda M, Takeuchi H, Mizuno T and Suzumura A: GM-CSF increases LPS-induced production of proinflammatory mediators via upregulation of TLR4 and CD14 in murine microglia. J Neuroinflammation 9: 268, 2012.

18. Wu Y,Li W, Zhou C, Lu F, Gao T, Liu Y, Cao J,Zhang Y, Zhang Y and Zhou C: Ketamine inhibits lipopolysaccharide-induced astrocytes activation by suppressing TLR4/NF-kB pathway. Cell Physiol Biochem 30: 609-617, 2012.

19. Yao L, Kan EM, Lu J, Hao A, Dheen ST, Kaur C and Ling EA: Toll-like receptor 4 mediates microglial activation and production of inflammatory mediators in neonatal rat brain following hypoxia: Role of TLR4 in hypoxic microglia. J Neuroinflammation 10: 23, 2013.

20. Blanco AM, Vallés SL, Pascual M and Guerri C: Involvement of TLR4/type IIL-1 receptor signaling in the induction of inflammatory mediators and cell death induced by ethanol in cultured astrocytes. J Immunol 175: 6893-6899, 2005.

21. Mohajeri M, Sadeghizadeh $\mathbf{M}$ and Javan M: Pertussis toxin promotes relapsing-remitting experimental autoimmune encephalomyelitis in Lewis rats. J Neuroinflammation 289: 105-110, 2015.

22. Nayak A, Dodagatta-Marri E, Tsolaki AG and Kishore U: An insight into the diverse roles of surfactant proteins, SP-A and SP-D in innate and adaptive immunity. Frontiers in immunology 3 : $131,2012$.
23. Liu J, Hu F, Wang G, Zhou Q and Ding G: Lipopolysaccharideinduced expression of surfactant proteins $\mathrm{A} 1$ and $\mathrm{A} 2$ in human renal tubular epithelial cells. J Inflamm (Lond) 10: 2, 2013.

24. Furlan R, Cuomo C and Martino G: Animal models of multiple sclerosis. Methods Mol Biol 549: 157-173, 2009.

25. Files DK, Jausurawong T, Katrajian R and Danoff R: Multiple Sclerosis. Primary Care 42: 159-175, 2015.

26. Matsumoto Y, Ohmori K and Fujiwara M: Microglial and astroglial reactions to inflammatory lesions of experimental autoimmune encephalomyelitis in the rat central nervous system. J Neuroimmunol 37: 23-33, 1992.

27. Napoli I and Neumann H: Protective effects of microglia in multiple sclerosis. Exp Neurol 225: 24-28, 2010.

28. Lundgaard I, Osório MJ, Kress BT, Sanggaard S and Nedergaard M: White matter astrocytes in health and disease. Neuroscience 276: 161-173, 2014.

29. Awasthi S, Madhusoodhanan R and Wolf R: Surfactant protein-A and toll-like receptor-4 modulate immune functions of preterm baboon lung dendritic cell precursor cells. Cell Immunol 268: 87-96, 2011

30. Bhatti F, Ball G, Hobbs R, Linens A, Munzar S, Akram R, Barber AJ, Anderson M, Elliott M and Edwards M: Pulmonary surfactant protein a is expressed in mouse retina by muller cells and impacts neovascularization in oxygen-induced retinopathy. Invest Ophthalmol Vis Sci 56: 232-242, 2015.

31. Agrawal V, Smart K, Jilling T and Hirsch E: Surfactant Protein (SP)-A suppresses preterm delivery and inflammation via TLR2. PLoS One 8: e639990, 2013.

32. Kawai T and Akira S: Pathogen recognition with Toll-like receptors. Curr Opin Immunol 17: 338-344, 2005.

33. Holmskov U, Lawson P, Teisner B, Tornoe I, Willis AC, Morgan C, Koch C and Reid KB: Isolation and characterization of a new member of the scavenger receptor superfamily, glycoprotein-340 (gp-340), as a lung surfactant protein-D binding molecule. J Biol Chem 272: 13743-13749, 1997.

34. Sano H, Sohma H, Muta T, Nomura S, Voelker DR and Kuroki Y: Pulmonary surfactant protein A modulates the cellular response to smooth and rough lipopolysaccharides by interaction with CD14. J Immunol 163: 387-395, 1999. 